\title{
PHYSICAL AND CHEMICAL CHARACTERIZATION OF NEUROLOGICAL INJURY IN AN AVIAN MODEL OF PRIMARY GENERALIZED EPILEPSY
}

\author{
EDWARD KENDALL ${ }^{1}$, ZHAO GONG ${ }^{1}$, JOHN TUCHEK ${ }^{2}$ AND ANDRÉ OBENAUS ${ }^{1,3}$ \\ University of Saskatchewan, Departments of ${ }^{1}$ Medical Imaging and ${ }^{2}$ Pharmacology, 107 Hospital Dr., \\ Saskatoon, SK S7K 0N8. Loma Linda University, ${ }^{3}$ Department of Radiation Medicine, CSP A1010, Loma \\ Linda, CA 92354, USA \\ e-mail: ejk339@duke.usask.ca \\ (Accepted May 1, 2002)
}

\begin{abstract}
Purpose This study was undertaken to determine if the epileptic chicken phenotype exhibited unique physical and chemical neurological changes. Methods Quantitative ${ }^{1} \mathrm{H}-\mathrm{MRI} / \mathrm{MRS}$ measurements were performed on adult control ( $\mathrm{Rr}$ ) and epileptic (rr) chickens. The metrics included nuclear relaxation rate, diffusion rate and concentration of metabolites associated with neuronal cells. Comparisons were performed using Students $\mathrm{t}$ or Mann-Whitney tests according to the data distribution. Results Proton density measurements detected no significant difference in density among the cerebral hemispheres and optic lobes within the control and epi birds, nor between bird phenotypes. Thus, within experimental error it appears that brain tissue density is similar in adult control and epi birds. Volume calculation from images spanning the entire brain confirmed that a consistent feature of the epi genotype was megalencephaly. Nuclear relaxation (T2 ) values obtained for the control bird were within the normal variant range for neuronal tissue. However, $\mathrm{T}_{2}$ 's of the epi birds were significantly higher than that of the controls ((158 ms versus $\left.123 \mathrm{~ms}\right)$. Similarly, in the diffusion data there was no apparent hemispheric bias. The optic lobes of the control animals exhibited an apparent diffusion coefficient significantly lower than that found for the cerebrum (Table 2). Interestingly, this differential was not apparent in the epi birds. The control birds across all regions of interest exhibited an ADC significantly less than that of the epi birds. Quantitative ${ }^{1} \mathrm{H}-$ spectroscopy using tissue extracts collected immediately after sacrifice revealed phenotypic differences in lactate, glutamate, creatine and NAA but not GABA. A reduction in NAA was detected in the cerebrum of epi birds. This is in agreement with the generally held view that seizure activity causes loss of neurones, thus the loss of N-acetyl aspartate. HPLC data corroborated the spectroscopic NAA findings but also detected an increase in GABA concentration in the epileptic birds. Conclusion The physical changes observed are consistent with dilution of the interstitial fluid by edema or through cellular reordering within the epi brain. The concomitant reduction in excitable neuronal cells and the relative increase in GABA observed in biochemical analysis are consistent with a fundamental change in the cellular population.
\end{abstract}

Keywords: magnetic resonance imaging and spectroscopy, primary generalized epilepsy, water diffusion.

\section{INTRODUCTION}

The discovery in 1970 (Crawford, 1970) of a domestic fowl with an autosomal recessive mutation that resulted in high susceptibility to seizures added a valuable model for basic epilepsy research. Cognitive and other studies have increased confidence in using the avian brain as a model for mammalian neuropathology (Colombo and Broadbent, 2000). Some of the physiological characterization of the model has been completed (Johnson and Tuchek, 1987; Gervais-Fagou, 1996), but lacking is an understanding of the biophysical implications of recurrent seizures. In addition, since the introduction of this model, the research paradigm has shifted with a move towards non-invasive technologies as indicators of the in vivo condition. Lastly, in terms of therapy, MR imaging and spectroscopy are increasingly important in assessing biochemical responsiveness to pharmacological intervention.

The homozygous recessive epi phenotype displays primary generalised tonic-clonic seizure activity in response to light (and acoustic) stimulation. This activity develops in ovo where spontaneous EEG seizure discharges were recorded at embryonic day 17 and discharges in response to photic stimulus at 
day 20. Spontaneous discharges declined towards hatching and generalized motor seizures did not appear before hatching (Guy et al., 1995). Once hatched spontaneous discharges are not observed but seizures can be reliably induced with intermittent light stimulation (ILS) (Guy et al., 1995). The seizures consist of three distinct phases. At first there is an opisthotonic arching of the neck, next the bird staggers back extending its wings and finally loses balance completely, flaps its wings and displays tonic-clonic leg movements. A period of post-ictal depression follows the seizure where the bird appears drowsy and lethargic.

In the non-convulsive state the animals' electroencephalograms (EEG's) typically show high amplitude slow wave activity. The EEG's of normal hatchmates show lower amplitude, higher frequency activity. When the epileptic phenotype (epi) are stimulated with ILS their EEG pattern is dominated by bilateral spiking that intensifies until the onset of motor seizures. Entrainment of the brainwaves with the ILS and the development of generalised epileptiform activity that corresponds to tonic-clonic seizure are seen (Crawford, 1983). No evidence of focal seizure activity has been observed.

The epi gene also causes megalencephaly. Brains of epileptic chicks are larger at birth than those of matched hatch mates; this difference continues through to maturity (Johnson and Tuchek 1987). Comparison of epi and control (heterozogous) brains revealed no obvious histological abnormalities nor did the cyto-architecture of individual cells appear markedly different (George et al., 1990). Enlargement was asymmetric in that it was most marked in the telencephalon and present to a lesser degree in the cerebellum. The enlarged regions contained more neurones and these were significantly larger and less densely packed (George et al., 1990).

There is compelling evidence that large prosencephalic and mesencephalic areas are involved in the epileptic disease (Guy et al., 1995). In a series of elegant experiments using neuro-chimeras researchers showed that grafts using epi prosencephalic tissue produced chicks that expressed neck arch and loss of balance but did not convulse. When mesencephalic tissue alone was grafted, seizures did not develop. Carefully performed EEG experiments demonstrated that neurons of the wulst in the prosencephalon of epi's show characteristic spontaneous bursting activity typical of other epilepsy models. Wulst neurons in prosencephalic chimeras did not exhibit bursting in response to ILS, nevertheless the chimeras did exhibit typical decoupling and flattening of the EEG during stimulation. In mesencephalic chimeras where seizures did not develop, neurons in the optic tectum were highly sensitive to ILS. These observations are consistent with a requirement for genetically altered prosencephalic tissue for generation, and for genetically altered mesencephalic tissue for propagation of motor seizure activity.

While megalencephaly is not a common feature of human epileptics it has been observed (Jack, 1995). Hemimegaloencephaly has been noted in patients presenting with seizures, developmental delay and functional impairments. The enlargement generally implicates the ventricle system as well as the cortex (Jack, 1995).

To date the neurochemical analysis of these fowl has revealed no abnormality that accounts for the underlying epileptic condition (Gervais-Fagou, 1996). Cerebral hemispheres from adult epi fowl contain significantly lower levels of 5-hydroxytryptamine and dopamine and higher levels of norepinephrine than non-epileptics. However, various approaches to correcting these imbalances did not reduce seizure activity (Johnson et al., 1981). Analysis of $\gamma$ aminobutyric acid (GABA) was inconclusive in that adult birds had significantly elevated GABA concentration but equally ILS sensitive chicks did not. Receptor characteristics were similar in control and epi as were the cyclic nucleotide pools (Crawford, 1983). There is an indication, that acetyl cholinesterase activity is elevated and acetylcholine transferase activity reduced in brain homogenates (Johnson et al., 1981).

Finally, from a pharmacological perspective, the birds respond similarly to human epileptics. Common anti-convulsants used in the treatment of human grand-mal epilepsy, were found to be equally effective in preventing ILS-induced seizures in the epi strain (Johnson, 1981; Johnson and Tuchek, 1987). Benzodiazepines widely used in epilepsy syndromes are potent anti-convulsants in epileptic fowl. Thus from a behavioural, anatomical, histolocical, biochemical and pharmacological perspective these fowl are a reasonable model for grand mal epilepsy in humans.

Here we report our findings comparing the physical and biochemical characteristics of epileptic and non-epileptic chicken brains as visualized with largely non-invasive magnetic resonance technology. We postulated that recurrent seizures in the epi phenotype would result in measurable biophysical alterations consistent with neuronal cell injury. 


\section{METHODS}

\section{ANIMAL PREPARATION}

Adult birds from the genetic strain of Fayoumi fowl exhibiting the predisposition to seizure were obtained from the poultry breeding program at the University of Saskatchewan. The birds were housed in a vivarium, and sorted into epileptic and nonepileptic using ILS challenge then transferred to individual battery cages. The animal experiments were carried out following a detailed protocol approved by the Animal Care Committee of the University of Saskatchewan. Birds were imaged under gaseous anesthesia (4\% Flurane, Abbott Labs, Saint-Laurent, QC, Canada): the animals were induced in a small custom chamber at (flow rate 1, vaporizer 1.5), a tracheal canula was placed and the animals maintained under a light plane of anesthesia (1.5\% Flurane, flow rate 0.9 , vaporizer 0.9 ) for the imaging phase. The birds were recovered by halting the anesthesia and removing the tracheal tube as consciousness returned. Six animal per treatment were used.

\section{MAGNETIC RESONANCE IMAGING}

${ }^{1} \mathrm{H}$-images were collected using a Magnex 3Tesla research scanner equipped with a Surrey Medical Imaging Systems console (Oxford, UK) using a custom built (Morris Instruments, Ottawa Canada) quadrature $3 \mathrm{~cm}$ coil, operating at $130.4 \mathrm{MHz}$ for protons. Typically the field of view was $25 \mathrm{~mm}$, (TR 2900ms, TE 20 - $180 \mathrm{~ms}$, Slice thickness $1.5 \mathrm{~mm}, 10$ slices) and the flip angle adjusted to provide consistent contrast. Concentration and relaxation weighted images (proton density, and $\mathrm{T}_{2}$ ) were collected using standard spin-echo sequences optimized for the desired tissue contrast. Relative water concentration (a value correlated with tissue density) was determined by dividing the mean pixel intensity of the region of interest by the mean pixel intensity of a water standard in the same proton density image. This allowed comparison among datasets collected under slightly different tuning and matching conditions.

$\mathrm{T}_{2}$ maps containing pure relaxation information were generated for regions in the archestriatum, neostriatum, optic lobe from a series of eight slices increasingly weighted for $\mathrm{T}_{2}$ as previously described (Wall et al., 2000). In brief, the data for each pixel in the series was fit using non-linear least squares to the relaxation equation: $\left.\mathrm{M}(\mathrm{t})=\mathrm{M}_{\mathrm{o}} \mathrm{e}^{\mathrm{t} / \mathrm{T}}{ }_{2}\right)$. This was solved to provide a map of the relaxation coefficient $\mathrm{T}_{2}$.

Diffusion weighted images were collected using standard spin-echo sequences and applying a weighting (b-factor) of $1000 \mathrm{~s} / \mathrm{mm}^{2}$ normal to the plane of observation.

Diffusion maps were generated as previously described (Sarty and Kendall, 1996). In brief, ADC was determined by the equation: $\mathrm{ADC}=\ln (\mathrm{So} / \mathrm{Sn}) / \mathrm{b}$ where $\mathrm{Sn}$ is the mean intensity for a weighted $\left(b=1000 \mathrm{~s} / \mathrm{mm}^{2}\right)$ image and So is the mean intensity for the corresponding image where the weighting factor is set to 0 . ADC's were calculated for each pixel in the image generating a diffusion map. ADC's for regions of interest (ROI) were calculated as the mean of the ADC for all pixels in the specified area. Each region of interest contained at least 100 pixels.

Image analysis was performed for each animal on a single slice selected as having the maximum crosssectional area for each region of interest. Cheshire ${ }^{\mathrm{TM}}$ image processing software (Hayden Image Processing Group, Waltham, MA) was used to outline and analyze the ROI's that were confirmed by a second analyst (Fig. 1). The bilateral ROI's were placed in the left and right cerebrum and optic lobes. Analysis included the mean value ( $\mathrm{T}_{2}$ or $\mathrm{ADC}$ ) and its standard deviation over the region of interest.

Fig. 1. Relaxation rate and apparent diffusion coefficient values were calculated as means of the regions of interest using Cheshire ${ }^{\mathrm{TM}}$ software. Regions were first drawn on anatomical images then propagated to the maps.

Brain volume was calculated from a set of $\mathrm{T} 1$ images spanning the entire organ. The brain perimeter

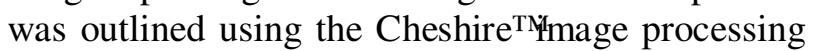
software to outline brain perimeter in each slice. Individual slice volumes were summed to compute 
the brain volume. Corrections were made for slice overlap based on the center to center distance of the slices.

\section{Spectroscopic analysis}

Tissue preparation. Intact brains were harvested from deeply anesthetized birds, quartered and flash frozen in liquid nitrogen. Once frozen the segments were ground to a fine powder in liquid nitrogen, weighed then freeze dried. An aliquot (approximately $140 \mathrm{mg}$ ) of the dried powder was removed from each sample for extraction and spectroscopic analysis. The aqueous extraction procedure followed the method of Peeling and Sutherland (Peeling and Sutherland, 1993). An internal standard, (3-trimethylsilyl) propionic-2,2,3,3- $\mathrm{d}_{4}$ acid (TMP, Aldrich, $75 \mu \mathrm{g} / \mathrm{sample}$ ) was added to determine losses during extraction and to provide a frequency reference. Extracts were freeze-dried a second time to remove water then taken up in $500 \mu \mathrm{l}$ of $\mathrm{D}_{2} \mathrm{O}$.

Spectroscopy. All spectra were collected using a Bruker $11.7 \mathrm{~T}$ magnet equipped with an AMX console operating at $500 \mathrm{MHz}$ for protons. Single pulse experiments (with a water suppressing pre-pulse) were used to collect relaxed spectra under the following operating conditions: one hundred and twenty scans with an inter-scan relaxation delay of 2 seconds (flip angle $60^{\circ}$ ) were collected for each sample providing a signal to noise ratio of at least 200 for the main peaks. The data were collected into 32768 time domain points for a frequency resolution of $0.24 \mathrm{~Hz} /$ point. $0.3 \mathrm{~Hz}$ line broadening was applied prior to Fourier transformation to the frequency domain.

Data Analysis. Peaks were classified based upon literature reference and on enrichment with authentic standards. Concentrations were determined from peak height which was corrected for isolation losses using the reference TMP peak and assuming a similar response for the TMP and sample protons.

concentration $=[$ peak height $/$ protons represented]/TMP height $] \times[75 \mu \mathrm{g} / \mathrm{sample}$ weight $]$

HPLC. Tissue was collected and processed as for spectroscopy. After the initial freeze drying, the soluble component was extracted by suspending the powder in ice-cold $75 \%$ ethanol for 30 min with intermittent vortexing. The particulate matter was removed by centrifugation (top speed in a Beckman microfuge) and the supernatant diluted with 2 parts $0.05 \mathrm{M}$ sodium acetate. Ten minutes prior to on column injection the sample was derivatized at room temperature by adding ortho-pthaldialdehyde reagent
(Sigma Chemicals). Sample concentration was determined by direct comparison with known standards.

Statistical analysis. Means from normally distributed data were compared using the standard Students t-test. In those cases where the data was not normally distributed the Mann-Whitney rank sum correlation test was used.

\section{RESULTS}

Proton density images $(\mathrm{TR}=2900 \mathrm{~ms}, \mathrm{TE}=20 \mathrm{~ms}$ ) provided optimum anatomical resolution for region of interest definition and volume studies. The primitive avian brain offers few well defined landmarks within the cerebral hemispheres and the optic lobes (Fig. 2A, B, C.), however the epi phenotype (Fig. 2D, E, F) was was associated with relatively large brain volume (cerebrum and optic lobes, $21 \pm 0.82 \mathrm{~mm}^{3}$ ) compared to a matched adult control $\left(16.66 \pm 1.37 \mathrm{~mm}^{3}\right.$, $\mathrm{p}<0.05)$.

Proton density images also by definition provide a direct, if somewhat insensitive measure of water concentration in the tissue. Under the fully relaxed conditions employed in this study, the signal intensity correlates with water concentration. Using this approach, we examined the relative tissue density of the chicken phenotypes within the regions of interest defined in Fig. 1. Relative tissue density was estimated by calculating mean pixel intensity over the region of interest and dividing this by the value from the mean intensity of a water phantom. The data in Table 1 indicate that these measures detected no significant difference in tissue density among regions analysed within control and epi birds, nor between bird phenotypes. Thus, within experimental error it appears that brain tissue density is similar in adult control and epi birds.

Bright regions in $T_{2}$ weighted images reflect the presence of bulk water, that is water that contains relatively few solutes (Fig. 2A, D). Thus, ventricles are relatively bright in $T_{2}$ images. If a series of images are collected with increasing $\mathrm{T} 2$ weighting the data can be fit to a curve to generate a $T_{2}$ map as described under Materials and Methods. These maps contain a single weighting, thus a $\mathrm{T}_{2}$ map contains no proton density information. The maps were subjected to region-of-interest analysis (Fig. 1) to determine mean apparent $\mathrm{T}_{2}$ coefficients. Each region of interest contained a minimum of 100 pixels. $\mathrm{T}_{2}$ data (Table 2) obtained from control and epileptic birds showed no significant differences among the regions of interest within a phenotype. 
Fig. 2. Coronal MRI images of Control (top) and epileptic (bottom) adult chickens. A,D $T_{2}$ weighted; B,E diffusion weighted; $C, F$ diffusion map. Images show the cerebral hemispheres and the optic lobes.

Table 1. Relative proton concentration of defined regions in the chicken phenotypes.

\begin{tabular}{l|c|c|c|c}
\hline \multirow{2}{*}{} & \multicolumn{4}{|c}{ Proton concentration relative to Water (1.0) } \\
\cline { 2 - 5 } & Left Cerebrum & Right Cerebrum & Left Optic Lobe & Right Optic Lobe \\
\hline Control & $0.91 \pm 0.13 \mathrm{a}$ & $0.83 \pm 0.10 \mathrm{a}$ & $0.88 \pm 0.12 \mathrm{a}$ & $0.89 \pm 0.13 \mathrm{a}$ \\
Epi & $0.86 \pm 0.12 \mathrm{a}$ & $0.87 \pm 0.12 \mathrm{a}$ & $0.84 \pm 0.14 \mathrm{a}$ & $0.82 \pm 0.15 \mathrm{a}$ \\
\hline
\end{tabular}

*Relative proton concentration was calculated by dividing mean pixel intensity of the region of interest by the mean pixel intensity of a water phantom. The image data were collected under fully relaxed conditions. Regions of interest are defined in Fig. 1. Values in a row or column followed by a different letter are significantly different at the 5\% level according to Students T-test.

Table 2. Comparison of $T_{2}(\mathrm{~ms})$ values obtained for each region of interest in adult control and epileptic birds.

\begin{tabular}{l|c|c|c|c|c|c}
\hline & $\begin{array}{c}\text { Cerebrum } \\
\text { mean }\end{array}$ & $\begin{array}{c}\text { Left } \\
\text { Cerebrum }\end{array}$ & $\begin{array}{c}\text { Right } \\
\text { Cerebrum }\end{array}$ & $\begin{array}{c}\text { Optic lobe } \\
\text { mean }\end{array}$ & $\begin{array}{c}\text { Left Optic } \\
\text { lobe }\end{array}$ & $\begin{array}{c}\text { Right Optic } \\
\text { lobe }\end{array}$ \\
\hline Control & $123.2 \pm 15.7 \mathrm{a}$ & $127.2 \pm 15.0 \mathrm{a}$ & $119.2 \pm 17.4 \mathrm{a}$ & $120.1 \pm 15.5 \mathrm{a}$ & $121.8 \pm 16.9 \mathrm{a}$ & $118.5 \pm 16.4 \mathrm{a}$ \\
Epileptic & $164.8 \pm 19.8 \mathrm{~b}$ & $161.7 \pm 6.6 \mathrm{~b}$ & $167.9 \pm 23.7 \mathrm{~b}$ & $153.7 \pm 18.1 \mathrm{~b}$ & $160.1 \pm 14.9 \mathrm{~b}$ & $149.3 \pm 20.6 \mathrm{~b}$ \\
\hline
\end{tabular}

$\mathrm{T}_{2}$ values were computed for each pixel in the series using non-linear least squares fit to the relaxation equation: $\mathrm{M}(\mathrm{t})=$ $\mathrm{M}_{\mathrm{o}} \mathrm{e}^{-\mathrm{t} / \mathrm{T}}{ }_{2}$ ). Mean values reported (ms) were determined as an un-weighted average over the region of interest. Values in a row or column followed by the same letter are not significantly different at the 5\% level according to a Students T-test. 
The values obtained for the control bird (119 $127 \mathrm{~ms}$ ) were within the normal variant range for mammalian neuronal tissue. However, $\mathrm{T}_{2}$ values of the epi birds (149 - $167 \mathrm{~ms}$ ) were significantly higher than those of controls for all the regions examined.

The $\mathrm{T}_{2}$ data suggested that the composition of the aqueous phase was different in Control and epi birds. We next investigated if the physical characteristics of the water were changed in the epi phenotype. Water diffusion imaging has been used extensively to detect regional cellular disruption associated with acute ischemic injury. Here were compared the apparent diffusion coefficients of control and epi birds.

Inspection of diffusion weighted images (Figs. 2B, E) revealed no discrete lesions, therefore a quantitative approach was adopted to assess water diffusion in control and epi brains. Diffusion maps were computed from a diffusion dataset using two " $b$ " values $(0$, $1000 \mathrm{~mm}^{2} / \mathrm{s}$, Fig. 2C, F). The maps were subjected to region-of-interest analysis (Fig. 1) to determine mean apparent diffusion coefficients (ADC). Each region of interest contained a minimum of 100 pixels and the diffusion encoding was normal to the plane of observation.

As with the $T_{2}$ data no hemispheric bias was detected. The optic lobes of the control animals exhibited an apparent diffusion coefficient significantly lower than that found for the cerebrum (Table 3). Interestingly, this differential was not apparent in the epi birds. The control birds across all regions of interest exhibited an ADC significantly less than that of the epi birds. A increased ADC results from an decrease in matrix viscosity or tortuosity.

The ADC values reported here contain contributions from both intra- and extra-cellular fractions. Where the extracellular fraction dominates the ADC value is higher; when the intracellular component dominates the ADC value is lower (van der Toorn et al., 1996; Lin et al., 1997). Relative changes in the ADC thus reflect the varying contributions of these components as well as changes within each of these compartments. Following this rationale, the epi birds appear to have experienced a general increase in interstitial space. The larger neurons noted by George and colleagues (Jack, 1995) may result in reduced cell packing, producing the shift to increased ADC.

We questioned if this remodeling would be reflected as a change in chemical composition. To test this we determined the concentrations of important amino acids in brain extracts using ${ }^{1} \mathrm{H}$ spectroscopy and HPLC.

A typical ${ }^{1} \mathrm{H}$-spectrum from a crude extract of the epi cerebrum demonstrates that many of the metabolites of interest were detectable, including GABA $(\gamma$ aminobutyric acid), GLUx (glutamate and glutamine), NAA (N-acetylaspartatic acid) and lactate (Fig. 3).

Differences $(\mathrm{p} \leq 0.05)$ between control and epi were detected for lactate $(\Delta=35 \mu \mathrm{g} / \mathrm{gfw})$, glutamate ( $\Delta=50 \mu \mathrm{g} / \mathrm{gfw})$, creatine $(\Delta=79 \mu \mathrm{g} / \mathrm{gfw})$ and NAA ( $\Delta=33 \mu \mathrm{g} / \mathrm{gfw}$ ) between the cerebral regions of interest of controls and epileptics. GABA concentrations did not differ significantly between control and epi phenotypes (Table 4). Creatine, alanine and lactate were reduced in epi birds. That lactate levels were lower was surprising in that it is often used as a marker for tissues under stress. NAA levels were reduced in the cerebrum and optic lobes of epi birds. This is in agreement with the generally held view that seizure activity causes selective loss of neurones, thus the loss of $\mathrm{N}$-acetyl aspartate.

Table 3. Comparison of $A D C\left(\mathrm{~mm}^{2} / \mathrm{s}\right)$ values obtained for each region of interest in adult control and epileptic birds.

\begin{tabular}{l|c|c|c|c}
\hline & $\begin{array}{c}\text { Left optic lobe } \\
\text { Mean }\end{array}$ & $\begin{array}{c}\text { Right optic lobe } \\
\text { Mean }\end{array}$ & $\begin{array}{c}\text { Left Cerebrum } \\
\text { Mean }\end{array}$ & $\begin{array}{c}\text { Right Cerebrum } \\
\text { Mean }\end{array}$ \\
\hline Control & $74.57 \pm 2.78 \mathrm{a}$ & $70.18 \pm 5.06 \mathrm{a}$ & $81.21 \pm 2.55 \mathrm{c}$ & $79.16 \pm 2.55 \mathrm{c}$ \\
Epileptic & $92.48 \pm 2.24 \mathrm{~b}$ & $88.71 \pm 2.28 \mathrm{~b}$ & $91.74 \pm 2.66 \mathrm{~b}$ & $89.13 \pm 2.81 \mathrm{~b}$ \\
\hline
\end{tabular}

$\mathrm{ADC}\left(\mathrm{mm}^{2} / \mathrm{s}\right)$ for each image pixel was determined by two-point fit to the equation: $\mathrm{ADC}=\ln (\mathrm{So} / \mathrm{Sn}) / \mathrm{b}$ where $\mathrm{Sn}$ is the mean intensity for a weighted $\left(b=1000 \mathrm{~s} / \mathrm{mm}^{2}\right)$ image and So is the mean intensity for the corresponding image where the weighting factor is set to 0 . ADC's were calculated for each pixel in the image generating a diffusion map. The reported ADC's for regions of interest (ROI) were calculated as the mean of the ADC for all pixels in the specified area. Each region of interest contained at least 100 pixels. Values in a row or column followed by the same letter are not significantly different at the $5 \%$ level according to a Students T-test. 

Table 5. Brain metabolites after extraction of cerebrum and optic lobe segments of control and epileptic birds determined using HPLC.

\begin{tabular}{l|r|r|r|r}
\hline \multirow{2}{*}{ Metabolite } & \multicolumn{3}{|c}{ Metabolite concentration (nmoles/mg protein) } \\
\cline { 2 - 5 } & \multicolumn{2}{|c}{ Cerebrum } & \multicolumn{1}{c}{ Optic Lobes } \\
\cline { 2 - 5 } & Control & $3.41 \pm 0.71 \mathrm{~b}$ & Control & Epi \\
\hline GABA & $2.47 \pm 0.39 \mathrm{a}$ & $2.79 \pm 0.37 \mathrm{~b}$ & $3.81 \pm 0.61 \mathrm{c}$ & $4.09 \pm 0.74 \mathrm{~d}$ \\
Aspartate & $3.18 \pm 0.42 \mathrm{a}$ & $11.74 \pm 0.87 \mathrm{~b}$ & $8.14 \pm 0.42 \mathrm{c}$ & $3.69 \pm 0.47 \mathrm{c}$ \\
Glutamate & $12.67 \pm 0.91 \mathrm{a}$ & $7.28 \pm 1.92 \mathrm{a}$ & $5.91 \pm 1.76 \mathrm{~b}$ & $5.84 \pm 0.44 \mathrm{c}$ \\
Glutamine & $8.23 \pm 1.48 \mathrm{a}$ & &
\end{tabular}

Values reported were determined by direct comparison to authentic standards. Values in a row followed by the same letter are not significantly different at the $5 \%$ level according to the Students t-test.

The HPLC based analysis of resected tissue provided further details. A reduction in NAA (reported as aspartate) was observed for the cerebrum regions, but not for the optic lobes. HPLC detected an increase in GABA concentration in the epileptic birds. This is in contrast with the spectroscopic data where no significant difference was obtained for GABA and where a reduction in NAA was observed in the optic lobes. It is possible that this discrepancy arises from the different normalization factors (gfw versus $\mu$ g protein) used in the analyses.

\section{DISCUSSION}

This is the first study to characterize a model of primary generalized epilepsy using magnetic resonance imaging and spectroscopy. The brains of adult epileptic birds were examined to determine if significant physical changes in neuronal tissue were associated with the phenotype. Our principal findings were: 1. Adult epileptic (rr) birds brains were significantly larger than those of control (Rr) hatchmates; 2. Proton density measurements indicated that epileptic and control birds had similar brain density; 3. Relaxation rate measurements suggested that the aqueous component is more dilute in epileptics than in control birds; 4. The apparent diffusion coefficient in epileptic birds was greater than that of control birds suggesting a reduction in cell packing density and or in matrix viscosity.

Measurements were performed both in vivo and in vitro. Brain volumes were computed by integrating MRI cross-sectional slice areas over the entire brain. Our data support the earlier findings of George et al. (1990) who reported that post mortem brains of epileptic birds were significantly larger than those of non-epileptic hatch-mates. Three measures of biophysical change were employed: relaxation weighted MRI, diffusion weighted MRI and magnetic resonance spectroscopy.
Relaxation weighted MRI is sensitive to those changes that alter the dielectric concentration of the matrix. Typically regions that experience vasogenic edema appear relatively bright in $\mathrm{T}_{2}$ images. Also, intracellular $T_{2}$ values are smaller in magnitude than those of the interstitial fluid (van der Toorn et al., 1996). The apparent $T_{2}$ values obtained in these experiments consist of contributions from both the interstitial and intracellular compartments.

Cell swelling from water migration into the cell will produce an increase in apparent $T_{2}$ of the intracellular component but will be accompanied by a reduction of the interstitial spaces and thus its contribution to $\mathrm{T} 2$. The overall apparent $\mathrm{T}_{2}$ from the tissue may decline or remain unchanged depending on the relative compartment volume. Since the apparent $\mathrm{T}_{2}$ increases in the epi birds, the changes observed were consistent with dilution of or a relative increase in the interstitial fluid.

Dilution is achieved by edema or by chemical modification of the matrix. Large scale dilution seems unlikely in that, the proton density measurements did not indicate an overall change in the bulk density of the tissue. This suggests that changes observed in the epi birds may be due to subtle changes rather than the profound regional edema that accompanies extensive infarct. These arguments rationalizing the increase in $\mathrm{T}_{2}$ generally favor moderate dilution of the tissue matrix. None of the imaging techniques provided visual evidence of focal lesions in the cerebrum or optic lobes, nor was there evidence of necrosis.

The $\mathrm{T}_{2}$ relaxation data comparing cerebral hemispheres and optic lobes indicated that the bulk water component within either the control or epileptic birds is similar (Table 2). This argues against large scale vasogenic edema such as is seen in acutely infarcted regions. However, the epileptic birds exhibited $\mathrm{T}_{2}$ values significantly greater than those of controls in all the regions of interest (Table 2). Considering that the proton density data suggested 
similar water (and conversely tissue) density, the $\mathrm{T}_{2}$ findings point to a shift in the water domain consistent with an increase in extra cellular space. Contributions from the dilute interstitial fluid increase the regionally averaged $\mathrm{T}_{2}$. The data ague against profound vasogenic edema since, while the $T_{2}$ would increase, the total water content would also increase. This was not observed in proton density measurements a generally reliable predictor of water concentration (Lin et al., 1997). Thus, the relaxation MRI measurement corollary is that while the neuronal tissues of control and epileptic birds have a similar cellular density the water populations have distinct features. We speculate that this could be achieved with a qualitative change in the cell population.

The epi birds exhibited brain enlargement or megalencephaly, our cross-sectional area measurements (Table 1) and the post-mortem findings of George and colleagues (George et al., 1990) indicate that epi brains are $25 \%$ greater in volume than control. They attributed the increase to both larger neuronal cells and a reduction in cellular packing. The proton density and relaxation data presented here argue against cellular swelling (which would decrease the apparent $T_{2}$ ) or increased vacuolation which would increase the proton density as causative. Again, the data are consistent with a qualitative change in the water component or in the cellular population.

Diffusion MRI measurements probe the physical environment of the reporter molecule. Changes that impact upon a molecular mobility will alter its diffusion characteristics measured here as apparent diffusion coefficient. These changes include matrix viscosity and tortuosity. For example, a reduction in extracellular space would be reflected as a decrease in the apparent diffusion coefficient due to increased interaction with reflective barriers.

The ADC values of the epi birds cerebrum and optic lobe tissues were significantly higher than those of age matched controls (Table 3). Thus, the data argue for a model where the neuronal tissue is: less densely packed and the soluble component less viscous. The $T_{2}$ data reported above argue in favor of a significant reduction in viscosity. However, this does not preclude contributions due to a change in the cellular composition or organization of the tissue. The diffusional changes were evidenced to a lesser extent in the cerebrum than in the optic lobes of epi birds. Taken together these changes are consistent with a mechanism where seizure injury occurs first or to a greater extent in the optic lobes and next or to a lesser extent in the cerebrum.
Clearly, the epileptic birds have experienced modification of their neuronal tissue. Consistent observations on loss of n-acetyl aspartate acid indicate that at a minimum there has been loss of neuronal cells. It is possible that, as the HPLC data suggest, the inhibitory cells remaining are hyperactive. All the changes noted (physical and biochemical) were widespread in that they were detected over large regions of interest. This is consistent with the work of Guy and colleagues (Guy et al., 1995) who determined that large mesencephalic and prosencephalic regions were implicated in the initiation and propagation of seizures in the birds.

Some human epilepsies have a genetic origin (Andermann, 1982) although the underlying physiology is poorly understood. This model is thus an attractive vehicle for investigating the biochemical origins of these seizure disorders. It may also prove useful for examining the developmental implications of recurrent seizures and the relationship between seizure frequency and neuronal competency. Others have reported that the avian brain is a useful if somewhat distant homologue of the mammalian brain (Colombo and Broadbent, 2000).

This report established that the adult epileptic bird has experienced significant biophysical modification of its neuronal tissue. These changes were consistent with a shift in the organization or composition of the cellular cohort in the regions of interest examined. It remains to be determined if the degree of cellular dysfunction correlates with seizure frequency.

\section{ACKNOWLEDGEMENTS}

The authors acknowledge the technical assistance of Jennifer Hadley, the financial support of the Health Sciences Utilization and Research Commission of Saskatchewan through a grant to EK and the support of MRV Systems Inc. in providing access to the MRI.

\section{REFERENCES}

Andermann E (1982). In: Anderson V, Hauser W, Penry J, Sing C Raven, eds. Genetic Basis of the Epilepsies. New York, pp. 355-74. cf Teillet M, Naquet R, LaSalle G, et al. (1991) Transfer of genetic epilepsy by embryonic brain grafts in the chicken. Proc Natl Acad Sci 88:6966-70.

Colombo M, Broadbent N (2000). Is the avian hippocampus a functional homologue of the mammalian hippocampus? Neuroscience and Biobehavioral Reviews 24:465-84.

Crawford RD (1970). Epileptic seizures in domestic fowl. J Hered 61:185-8. 
Crawford RD (1983). Genetics and behavior of the epi mutant chicken. In: Olkawa $\mathrm{T}$, ed. The brain and behaviour of the fowl. Tokyo, Japan: Scientific Press, 259-69.

George DH, Munoz DG, McConnell T, Crawford RD (1990). Megaencephaly in the epileptic chicken: a morphometric study of the adult brain. Neuroscience 39(2):471-7.

Gervais-Fagou D (1996). Learning impairment and the cholenergic system in epileptic fowl. PhD Thesis. University of Saskatchewan, 101-115.

Guy NTM, Fadlallah N, Naquet R, Batini C (1995). Development of epileptic activity in embryos and newly hatched chicks of the Fayoumi mutant chicken. Epilepsia 36(1):101-7.

Jack CR (1995). Epilepsy: Magnetic Resonance Imaging, neuroimaging and anatomy. Neuroimaging Clinics of North America 5(4):597-622.

Johnson D, Tuchek J (1987). The epileptic chickens: In Jobe P, Laird H, eds: Neurotransmitters and epilepsy. Clifton NJ: Humana Press, 95-114.

Johnson DD, Jaju A, Ness L, Richardson JS, Crawford RD (1981). Brain norepinephrine, dopamine, and 5- hydroxytryptamine concentration abnormalities and their role in high seizure susceptibility of epileptic chickens. Can J Physiol Pharmacol 59:144-9.

Lin W, Pazcynski R, Venkatesan R, He Y, Hsu C, Haake E (1997). Quantitative brain water measurements with magnetic resonance imaging in a focal ischemia model. Magn Reson Med 38:303-10.

Peeling J, Sutherland G (1993). ${ }^{1} \mathrm{H}$ magnetic resonance spectroscopy of extracts of human epileptic neocortex and hippocampus. Neurology 43:589-94.

Sarty G, Kendall E (1996). Self-diffusion maps from wavelet de-noised images. J Magn Reson Series B. 111:50-60.

van der Toorn A, SykováE, Dijkhuizen RM, et al. (1996). Dynamic changes in water ADC, energy metabolism, extracellular space volume, and tortuosity in neonatal rat brain during global ischemia. Magn Reson Med 36:52-60.

Wall C, Kendall E, Obenaus A (2000). Rapid alterations in apparent diffusion coefficients with anatomical correlates in a rodent model of status epilepticus. Am J Neurorad 21:1841-52. 